\title{
A Theoretical Basis for Using Virtual Worlds as a Personalised Process Visualisation Approach
}

\author{
Hanwen Guo, Ross Brown, and Rune Rasmussen \\ Information Systems School, Science and Technology Faculty, \\ Queensland University of Technology, 2 George St, Brisbane, 4000, Queensland, Australia \\ \{h1.guo, r. brown, r. rasmussen\} @qut. edu. au
}

\begin{abstract}
Communication processes are vital in the lifecycle of BPM projects. With this in mind, much research has been performed into facilitating this key component between stakeholders. Amongst the methods used to support this process are personalized process visualisations. In this paper, we review the development of this visualization trend, then, we propose a theoretical analysis framework based upon communication theory. We use this framework to provide theoretical support to the conjecture that $3 \mathrm{D}$ virtual worlds are powerful tools for communicating personalised visualisations of processes within a workplace. Meta-requirements are then derived and applied, via 3D virtual world functionalities, to generate example visualisations containing personalized aspects, which we believe enhance the process of communication between analysts and stakeholders in BPM process (re)design activities.
\end{abstract}

Keywords: Business Process Management, 3D Virtual Worlds, Process Communication.

\section{Introduction}

Presently, Business Process Management (BPM) is recognized as a set of approaches that can facilitate business activity management [1], and its lifecycle involves four phases, which are design, configuration, enactment and diagnosis [2]. In these four phases, the communication process has been recognized as a vital component, since it is one factor, among others, that is highly related to the success of BPM projects [3].

To enhance the communication process, visual assistance approaches have been employed. Conceptual diagrams, such as BPMN [4] or ER diagrams [5], are shown to other stakeholders by business analysts, when complex business environments need to be investigated. Despite these approaches, it is reported that business analysts and stakeholders often have communication problems [6-8]. This is because we cannot guarantee that every stakeholder has the necessary process diagram knowledge to understand what is being presented [9].

A 3D virtual world is a network-based, computer synthesized dynamic environment, where participants can communicate with each other and interact with rich 3D computer-generated environmental objects [10]. Recently, it has been realized that such 3D virtual worlds can be applied in observational ethnography, case 
studies, economic markets and social networks [10]. Its richer visualization abilities can expand the memory bandwidth of people, allowing them to effectively process more information [11]. This strongly suggests that 3D virtual worlds could be a superior process visualization platform, enabling people to recall and cognate about conceptual and non-conceptual content. Such an approach may facilitate communication processes in analysing, modelling and validating organizational structures, and related resource behaviours. In addition, these visualization have the capacity for high levels of personalized configuration, providing role-based representations that enable people to recognize their place in a complex process system, via provision of a visual context simulating their own experience [45].

As a new research area, researchers are working on BPM oriented 3D virtual world applications [12-17]. Despite the fact that these works are available and can be recognized as visualization approaches for enhancing communication approaches, theory-based analysis outputs are still lacking. Theory analysis in Information Systems or BPM domains, which is conducted ahead of system developments, can provide guidance that caters to needs in reality [18]. In other words, the lack of theory analysis may impede us in developing sophisticated 3D visualization systems for BPM in the future.

With these points in mind, we propose a theory-based framework and utilize this framework to analyse problematic areas in communication processes, explain why problems occur, predict how to overcome these problem and suggest how to design an effective communication approach within a BPM context.

This paper is organized as follows: Section 2 discusses related work. Section 3 explores the capabilities of using a virtual world as a communication approach. Section 4 provides a mapping of virtual world capabilities to personalized visualizations. And at last, Section 5 concludes with a discussion of achievements, and points towards future work.

\section{Related Work}

In 2008, we proposed a BPM oriented virtual world application [13], where we claimed that 3D virtual worlds can be utilized as a communication approach in the BPM context. Then, we proposed an extended business animation system [12]. Following these initial efforts, other researchers have published BPM oriented virtual world applications. Perkins [14] advocates that intelligent agent systems should be integrated for demonstrating designed business plans. Chodos et al. [15] have proposed a workflow based simulation system, named MeRiTS, for professional medical training. These works have sought to visualize the operational levels of a business process environment, typically for training applications in the health domain, see Fig. 1. However, along with other commercial systems, such as Flexsim ${ }^{1}$, there is an absence of a focus on personalised forms of visualization, in particular, the overlaying of surrounding information aligned with the tasks being represented for a particular business role and its potential personalized variations.

${ }^{1}$ http: //www.flexsim.com/flexsim/ 

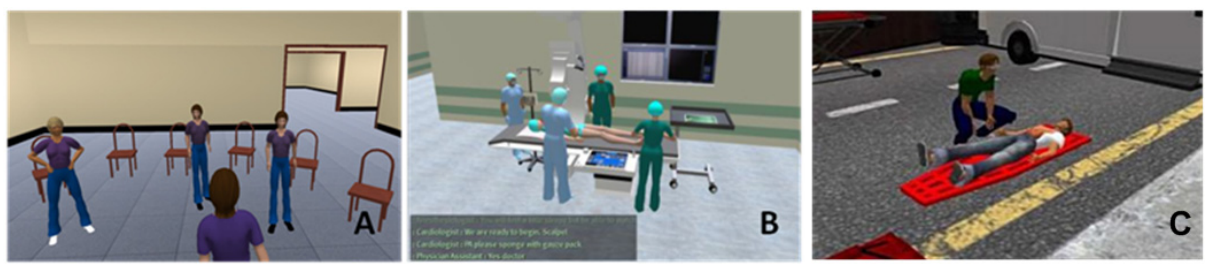

Fig. 1. Illustrations from previous work by [19] (picture A), [14] (picture B) and [15] (picture C)

One of the major positive findings with virtual worlds has been the quantitative results showing the superior capabilities of such technologies in knowledge transfer in training scenarios [46], or that they are at least equal to present physical methods [47]. This is consistent with theoretical models presenting a proposed hierarchy of simulation above other less interactive approaches, producing better learning outcomes via greater retention of information [48]. These promising initial educational results are highly relevant to process communication issues. Such capabilities in the transfer of knowledge can be seen as overlapping with activities in process redesign requiring clear communication of process knowledge. Evidence has emerged indicating that a more human interaction oriented approach may offer better communication results [41].

Personalised process model visualization and presentation continues to be a very active area of research [42- 45]. Personalized process model visualization modifies a base process model to conform to the specific information requirements of a stakeholder within a process model. Of particular interest is the insight that the use of iconic or pictorial representations of process activities may provide better insight into the process for a stakeholder [45]. Only preliminary evidence is available at the moment to show that this personalized view of a process actually increases understandability [44]. The question of the effectiveness of personalized views is yet to be fully answered in the affirmative.

At this moment in time, there is no research that we are aware of that has explored the possibility of using 3D virtual worlds as an environment for personalized process displays. Therefore, as we believe this is a first paper on this personalized topic, there is a need to provide theoretical analysis motivating such research. In the following, we will propose our analysis framework, with extensions to our previous work [20], in order to support our proposal that a 3D virtual world is a valid and effective approach to personalized process visualization.

\section{Framework Development}

Our intention in this section is to generate an appropriate analysis framework to support our conjectures regarding the efficacy of 3D virtual worlds for personalized process visualisations. Therefore, using an approach drawing from the popular theory development work of Gregor [18], and used in closely related visualization work [8], we propose an analysis framework with an objective to support, in theory, the argument that a virtual world can be used to improve communication between 
business analysts and stakeholders in a BPM communication context. The framework involves three major theory development theories from Gregor (Analysis, Explanation and Prediction, and Design and Action) that build on each other to support our conjecture. The analysis theory seeks to characterize the information transmission between business analysts and other stakeholders. The explanation and prediction theory seeks to explain related cognitive processes in the mind of business analysts and other stakeholders, predicting how to enhance them. A final design and action theory is used to support the conjecture that $3 \mathrm{D}$ virtual world affordances can support the developed theories on BPM communication.

\subsection{Analysis of the BPM Communication Model}

Many researchers have created conceptual models and theories for describing such communication. Among these conceptual models, the one proposed by Jakobson [21], Shannon and Weaver [6] has been widely used in different areas, such as information technology [22], software engineering [8] and media studies [23]. The common components in these two models are an information sender and receiver, information coding and transition processes, and factors that can affect the quality of information transition. They $[6,22]$ both believe good communication requires the sender and receiver to use the same code, while noise, as variations in the coding process, can slow down cognitive processes. The core ideas and components of their conceptual communication models has been adapted to represent communication between business analysts and other stakeholders in a BPM redesign context in Fig. 2. For this context, we concentrate on the business analyst and other stakeholder relationships as a first point of call. The stakeholders in our case are typically domain experts experienced with the processes in question, but not knowledgeable of process model grammars. For example, in a hospital management scenario, the stakeholder may be a nurse, doctor or an administrator.

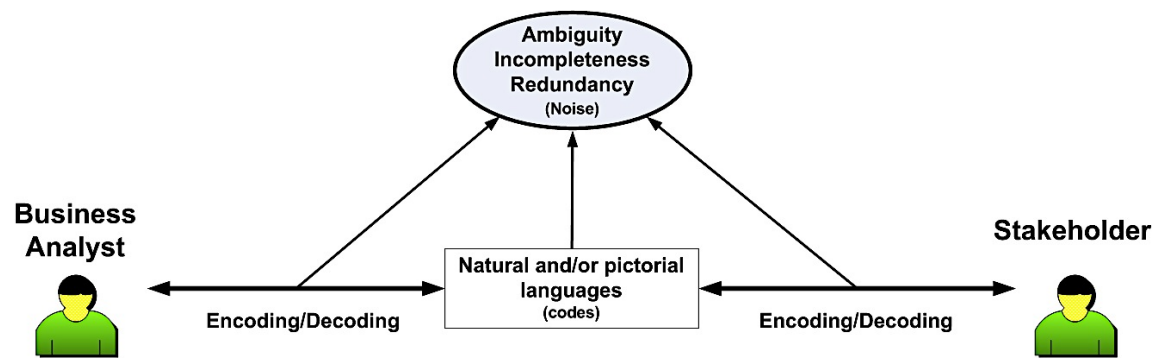

Fig. 2. Illustration of a modified communication model within the BPM context, with the business analyst and other stakeholder taking alternating sender and receiver roles. Codes in this case are defined as the pictorial languages used in process modeling, while noise has been defined for this context as ambiguity, incompleteness and redundancy in the process representation, leading to reported communication problems between analysts and other stakeholders [9]. 
In practice, the aim of the diagnosis phase in the BPM life cycle is to capture, analyse, automate and optimize present business processes. Issues such as, measuring the gap between current business processes and desired business processes, should be discussed in this phase [3, 24]. An accurate and mutual understanding between business analysts and stakeholders is therefore critical in decision making regarding process improvements [3].

On the one hand, business analysts need to gain a better comprehension of requirements and feedback from their stakeholders. This can help them design an improvement plan that suits the needs of their clients, and to continually revise the plan according to stakeholder preferences. On the other hand, stakeholders need to articulate their requirements so that business analysts can examine the validity of the newly designed business process model, to confirm that the improved and optimized business processes are appropriate, as proposed by the business analyst.

It is typical, during the design of a visualization approach, to include an analysis of the particular stakeholder tasks needing visual support [49]. We apply this approach to the analyst stakeholder interactions during a process validation activity. During their discussions about a business process, both business analysts and operational managers are interested in a number of topics, with some of them being: the specific sequence of task events, personnel arrangements and human resource behaviours at the operational level of a business environment [25-27]. A diagram showing the communication skill set of a business analyst, and the interests of a manager or client, are represented in Fig. 3, below.

So far, communication theories $[6,22]$ have been used to analyse the communication roles of business analysts and stakeholders, and the code forms they utilize. It can be said that business analysts and stakeholders alternatively play the role of message sender and receiver in the communication process.

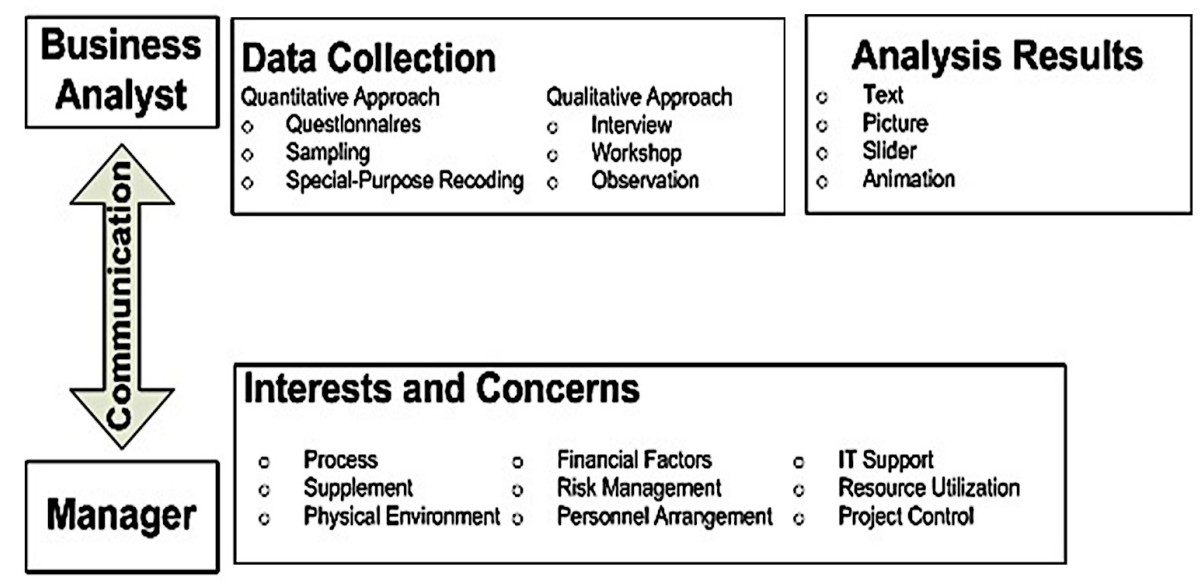

Fig. 3. Illustration of the main information concerns of business analysts and managers

From this previous research, two major conclusions can be reached. One, stakeholders cannot always represent their understanding and thoughts about business activities in a well structured way [7]. Their goal may not be clearly defined and a 
variety of unexpected factors can influenced their satisfaction with meeting results [28]. Two, the visual codes used by business analysts inevitably interfere with cognitive processes in the readers. To understand the visual codes requires a cognitive process within the stakeholder, to work on the conceptual model representations. The success of the cognitive process relies on long term memory that contains prior knowledge about the conceptual model, such as abstract model grammars and terminologies [8]. In practice, it cannot be expected that visual code readers, in particular domain specialists, have such prior knowledge. Such lack of knowledge leads to results showing that conceptual models puzzle domain specialists [7-9]. In part, this is due to the their inability to align the abstracted representation with their own personal and experiential understanding of the business process being discussed.

\subsection{Explanation and Prediction}

In the following, conjectures drawn from cognitive theories [11, 29-31] are made to explain why the noise occurs in the communication process and predicts what measures can reduce the noise to promote the efficiency of the communication process.

Explanation. Fig. 4. represents information processes in the human brain according to a popular model known as the Human Process Model (HPM) [32].

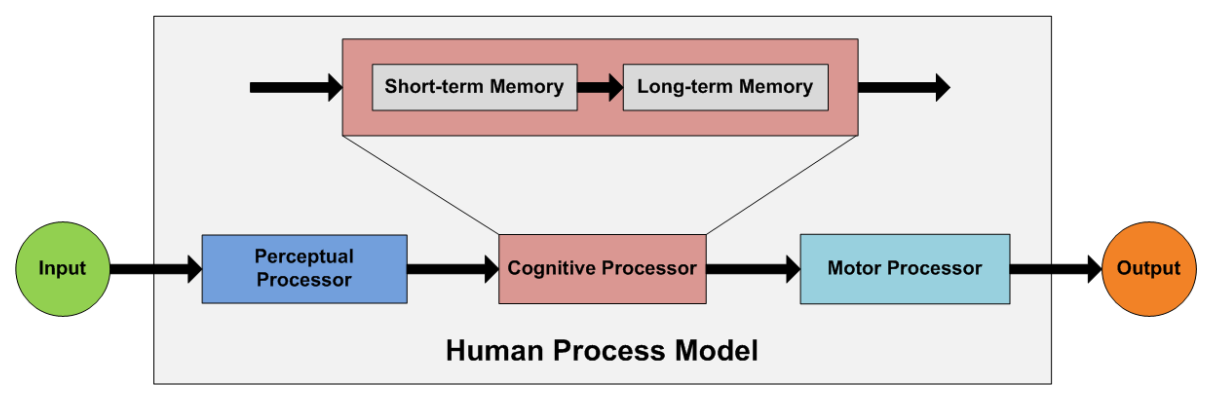

Fig. 4. Illustration of the Human Process Model that is adapted from [32]. The model describes the procedure that humans use to process information.

The Perceptual Processor models how people sense input information, such as a process model in a graphical representation. Sense organs, such as eyes and ears, receive input information, which will be passed to the Cognitive Processor.

The Cognitive Processor in the model, analyses the input information by utilizing short-term memory, which is a temporary storage area loaded with a stream of input signals. It is believed that the loading capability of such working memory is important in both verbal and visual information processing [33, 34].

Long-term memory permanently stores some prior knowledge. The amount of knowledge determines how a human responds to the input information [35]. If prior knowledge is relevant to the input information, the motor processor will receive positive indications, enabling a human to take a correct action. Otherwise, the motor processor will receive negative indications, giving an irrelevant or wrong answer. 
With reference to this model of information processing, it can be said that the cognitive processor plays an important role in problem solving $[29,36]$. In the context of this research, it can be said that the performance of communication between business analysts and stakeholders is dependent on this same cognitive process.

Therefore, a reverse argument can be made; the increase of loading capability in the short-term memory and presence of prior knowledge in the long-term memory can optimize and improve the cognitive processes involved, potentially improving communication between stakeholders in a meeting.

Prediction. According to cognitive load theory [30], humans have limitations in processing information, that is, humans usually can process seven, plus or minus two items without context [11]. This is a bottleneck with regards to communication improvement and optimization. Miller [11] states that one possible solution for communication improvement is to increase the loading capability of the short-term memory of the receiver, arousing prior knowledge in the longer-term memory to achieve better performance.

In BPM consultation practice, tools and techniques, such as individual or group interviews, questionnaires, conceptual models, workshops and prototypes, aim to achieve better communication [26, 28, 37]. These tools and techniques provide people with a wide range of communication methods.

The main conjecture of this paper is that personally configurable $3 D$ virtual world tools and techniques may increase the loading capability of the short-term memory and arouse prior knowledge in the longer-term memory, consistent with the arguments of Miller [11]. For example, an accurate visualization about the working environment may arouse the prior knowledge of a stakeholder. Many stakeholders work in a real environment, not a conceptual space. They are very visually familiar with their working environment. A personalised visualization of their working environment can provide them with a representation in a "hands-on" manner that simulates real artefacts in real spaces. This enables them to make comments according to their work experience.

Moody concludes that this "hands-on" manner is a form of semantic transparency [8]. Such a transparency provides the real meaning and appearance of conceptual models. People can directly infer information in the conceptual model from the operational representation of the conceptual models in a simulated workplace.

Indeed, these visualization capabilities can be combined and employed to inform an individual or group interview, questionnaire, conceptual model, workshop and prototype [26, 28, 37]. Therefore, these visualization needs can be recognized as meta-requirements for communication improvement and optimization, increasing the loading capabilities of the short-term memory to arouse prior knowledge in the longer-term memory.

\section{Effective Communication Approach Design}

An appropriate Design and Action Theory can guide us to systematically and scientifically design a better communication approach [18]. Two design theories proposed by Markus et al. [38] and Walls et al. [39] state that if system A can meet the meta-requirements for developing another system B, then system A can be 
adapted to facilitate the development of system B. Guided by such design theories $[38,39]$, the following sections will investigate the features of a $3 \mathrm{D}$ virtual world and then discuss the feasibility of utilizing 3D virtual worlds to satisfy previously mentioned meta-requirements.

Virtual world features can be used to create BPM oriented visualization applications, satisfying communication meta-requirements we have identified, facilitating the BPM Communication Model. The supporting relationship between aforementioned meta-requirements and features of a virtual world application are represented in Table 1.

Table 1. Discussion of the relationship between meta-requirements and virtual world features

\begin{tabular}{c|l}
\hline Meta-requirements & \multicolumn{1}{|c}{ Virtual World Features } \\
\hline Entity Representation & Geometry Representation \\
\hline Physical Environment & Geometry Representation \\
\hline User Participation & Interaction and Collaboration, Avatar \\
\hline Prototype Demonstration & Geometry Representation, Functional Configuration, \\
Information Display & Avatars, Behaviour Modelling \\
\hline Human Resource Behaviour & Functional Configuration, Information Visualization \\
\hline
\end{tabular}
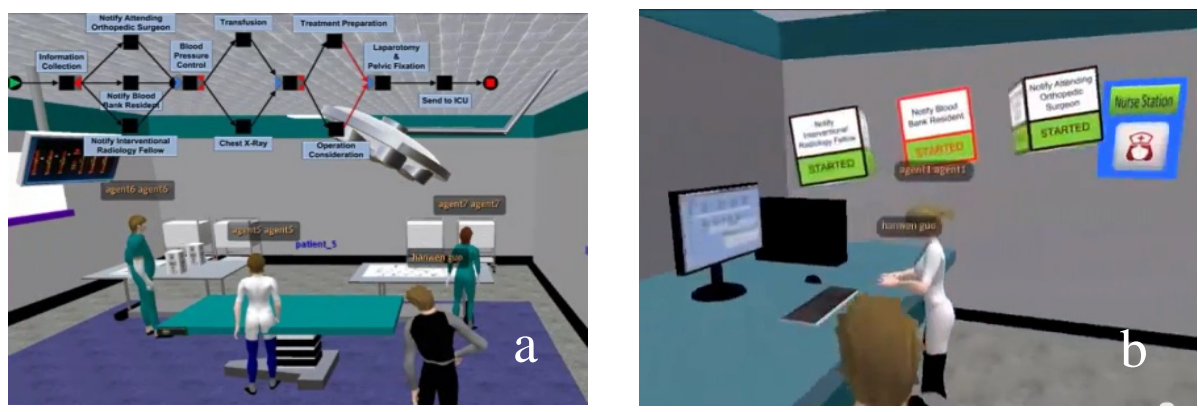

Fig. 5. Example personalized virtual world process visualisations. Firstly, the $2 D$ HUD (a) is a representation of the process model of interest, with its state highlighted using red annotations. The second (b) is a resource-aligned representation providing a role-oriented view of the tasks being specifically performed by the simulation agent within the view.

This process has driven the design of our series of process model simulations and visualisations that can be focused on a particular role within an organization. The visualisations are developed using an agent-based system we have created to simulate control and resource workflow patterns [20]. The agents are provided work using a role-based push approach from a business process system. We now detail the meta-requirements for such communication, and how this has motivated the virtual world visualization design. In particular, we focus on how this can personalize a 
visualization to assist understanding by a stakeholder. A series of selected features [40] and capabilities are now analysed. In each case, the examples referenced are those shown in Fig. 5, which illustrates a hospital admissions process we have visualized in a virtual world ${ }^{2}$.

Entity Representation. Business analysts typically use 2D shapes constrained by visual grammars to abstractly represent real objects, in particular, to describe the relationships between tasks and state transitions between tasks [4]. In the visualisations shown below, the YAWL process modeling grammar is used to annotate the scene to provide process model information. We show two examples of this annotation, via a 2D Heads Up Display (HUD) and a resource centric overhead display. This relates the abstract process activities to an operational environment, with agent representations of specific roles, to increase insight.

Physical Environment. In a 3D virtual world, a complex and realistic environment can be built up from basic geometries. Key physical objects related to processes and roles are depicted in the images as a basis for the visualization. In the case of our example, relevant medical equipment, such as beds, administration desks, amongst other items, are physically modeled in order to provide a representation of a familiar working environment, personalizing the visual to match the workplace of a viewer.

Human Resource Behaviour. Observers can obtain insight into the position level, nature of work, interpersonal relationship, intention and characteristics of an observed person performing work activities via their appearance [14, 25]. In a 3D virtual world, avatars or agents can be created that accurately model the work behaviour of people in reality. In the example shown, tasks have been allocated to agents according to a role model within the process, with their commensurate visual behaviours.

Prototype Demonstration. Business analysts can use some visualization approaches, such as a configurable simulation, to demonstrate a prototype [26, 28, 37]. Such visual assistance is an essential component of requirements elicitation and analysis, enabling business analysts and stakeholders to discuss unforeseen situations that may happen in their work. In a virtual world, a prototype can clearly demonstrate human resource behaviour, enabling stakeholders and business analysts to observe characteristics of the business plan via interactions between agents and objects in the virtual worlds. In a similar vein to configurable process simulation, the examples demonstrate, using multiple agents, each process instance in parallel.

User Participation. Users of virtual worlds can log into a virtual world from a remote location with a software client. The users who log into the virtual world will be represented as avatars, providing people with communication tools for engagement. The darker avatar in each image in the examples, is logged in remotely to the server, and can thus view the simulation as it is performed. Even a side-by-side analyst and stakeholder meeting can benefit from this interaction, as the logged in avatar provides an in-world gesturing device.

\footnotetext{
${ }^{2}$ Videos of visualizations are available at: https : / / www . youtube. com/watch? v=m6Losma61-U
} 
Information Display. Information may be loosely classified as qualitative and quantitative. These types of information reflect the temporal state of the business environment. Representations of this information can, amongst other things, provide people with insight into the workload of human resources and utilization rates of nonhuman resources. Our examples show, via a HUD, the display of the current state of the process model case being visualized, or resource centric information regarding the state of each agent in the simulation.

\section{Conclusion}

In this paper, a theoretical framework has been proposed for solving problems in communication between analysts and other process stakeholders. Firstly, it has utilized communication theories $[6,21]$ to identify the main components of communication between business analysts and stakeholders. Then, cognitive theories $[11,29,30]$ were used to explain why the performance of the communication process can be negatively affected by more abstract process model grammars. Metarequirements for communication improvement and optimization were identified. Finally, features of 3D virtual worlds were analysed, forming a mapping of their key features to communication requirements, showing how the features of a virtual world can be combined to assist the validation techniques used by a business analyst. Specific examples from a hospital virtual world we have implemented were used to illustrate the use of virtual worlds for personalized views. In particular, the views can be personalized to the operational view that a stakeholder has of their work.

This work is preliminary in nature, and has only begun to investigate possible approaches to providing 3D personalized visualisations. In future work, we will design an evaluation framework, which is based on the discussion in this paper, to evaluate the presented visualization approaches. In addition, the visualization interfaces will be updated to reflect the latest approaches to $2 \mathrm{D}$ personalized visualization [45], providing intuitive filtering and display approaches for a more fine-grained personalized experience of 3D process model information.

Acknowledgments. This research has been supported by Smart Service CRC in Australia (http://www.smartservicescrc.com.au).

\section{References}

1. Brocke, J.V., Rosemann, M. (eds.): Handbook on Business Process Management: Strategic Alignment, Governance, People and Culture. Springer, Heidelberg (2010)

2. van der Aalst, W.M.P., ter Hofstede, A.H.M., Weske, M.: Business Process Management: A Survey. In: van der Aalst, W.M.P., ter Hofstede, A.H.M., Weske, M. (eds.) BPM 2003. LNCS, vol. 2678, pp. 1-12. Springer, Heidelberg (2003)

3. Trkman, P.: The critical success factors of business process management. International Journal of Information Management 30(2), 125-134 (2010)

4. Wohed, P., van der Aalst, W.M.P., Dumas, M., ter Hofstede, A.H.M., Russell, N.: On the Suitability of BPMN for Business Process Modelling. In: Dustdar, S., Fiadeiro, J.L., Sheth, A.P. (eds.) BPM 2006. LNCS, vol. 4102, pp. 161-176. Springer, Heidelberg (2006) 
5. Davies, I., Green, P., Rosemann, M., Indulska, M., Gallo, S.: How do practitioners use conceptual modeling in practice? Data \& Knowledge Engineering 58(3), 358-380 (2006)

6. Shannon, C., Weaver, W.: The Mathematical Theory of Communication. University of Illinois Press, Chicago (1963)

7. Sadiq, S., Indulska, M., Bandara, W., Chong, S.: Major Issues in Business Process Management: A Vendor Perspective. In: 11th Pacific-Asia Conference on Information Systems: Managing Diversity in Digital Enterprises (PACIS 2007), pp. $40-47$ (2007)

8. Moody, D.: The "Physics" of Notations: Toward Scientific Basis for Constructing Visual Notations in Software Engineering. IEEE Transactions on Software Engineering 35(6), 756-779 (2009)

9. Khatri, V., Vessey, I., Ramesh, V., Clay, P., Park, S.: Understanding Conceptual Schemas: Exploring the Role of Application and IS Domain Knowledge. Information Systems Research 17(1), 81-99 (2006)

10. Bainbridge, W.: The Scientific Research Potential of Virtual Worlds. Science 317(5837), 472-476 (2007)

11. Miller, M.: The magical number seven, plus or minus two. Psychological Review 63, 8197 (1956)

12. Brown, R., Rasmussen, R.: Virtual Environment Visualisation of Executable Business Process Models. In: Rao, R. (ed.) Virtual Technologies for Business and Industrial Applications: Innovative and Synergistic Approaches, pp. 68-88. IGI Global Press, Hershey (2010)

13. Brown, R., Cliquet, F.: Communication of Business Process Models via Virtual Environment Simulations. BPTrends 12(9), 1-7 (2008)

14. Perkins, K.: Workflow Simulation in a Virtual World, Master Thesis in Computer Science and Computer Engineering Department, University of Arkansas, Fayetteville (2001)

15. Chodos, D., Stroulia, E., King, S.: MeRiTS: Simulation-Based Training for Healthcare Professionals. Studies in Health Technology and Informatics 163, 125-131 (2011)

16. Betz, S., Eichhorn, D., Hickl, S., Klink, S., Koschmider, A., Li, Y., Oberweis, A., Trunko, R.: 3D representation of business process models. In: MobIS, pp. $73-87$ (2008)

17. Eichhorn, D., Koschmider, A., Li, Y., Sturzel, P., Oberweis, A.: Trunko. R.: 3D support for business process simulation. In: 33rd Annual IEEE International Computer Software and Applications Conference, COMPSAC 2009, vol. 1, pp. 73-80 (2009)

18. Gregor, S.: The Nature of Theory in Information Systems. MIS Quarterly 30(3), 611-642 (2006)

19. Brown, R., Rasmussen, R., Baldwin, I., Wyeth, P.: Design and implementation of a virtual world training simulation of ICU first hour handover processes. Australian Critical Care Journal 25(1), 178-187 (2012)

20. Guo, H., Brown, R., Rasmussen, R.: Virtual worlds as a model-view approach to the communication of business processes models. In: CEUR Workshop Proceedings (2012)

21. Jakobson, R.: Closing statement: Linguistics and Poetics, in Style. In: Sebeok, T.A. (ed.) Language, pp. 350-377. MIT Press, Cambridge (1960)

22. Hollnagel, E., Woods, D.: Joint Cognitive Systems: Foundations of Cognitive Systems Engineering. CRC Press, Boca Raton (2005)

23. Manovich, L.: The language of new media. The MIT Press, Cambridge (2001)

24. Nah, F., Lau, J., Kuang, J.: Critical factors for successful implementation of enterprise systems. Business Process Management Journal 7(3), 286-296 (2001)

25. Lewis, M., Slack, N.: Operations Management: Critical Perspectives on Business and Management. Routledge, New York (2003)

26. Reid, R., Sanders, N.: Operations Management. Wiley, New York (2009) 
27. Voss, C., Tsikriktsis, N., Frohlich, M.: Case research in operations management. International Journal of Operations \& Production Management 22(2), 195-219 (2002)

28. McConnell, S.: Rapid Development: Taming Wild Software Schedules. Microsoft Press, Redmond (1996)

29. Newell, A., Simon, H.: Human Problem Solving. Prentice Hall, Upper Saddle River (1972)

30. Chandler, P., Sweller, J.: Cognitive Load Theory and the Format of Instruction. Cognition and Instruction 8(4), 293-332 (1991)

31. Flugel, J.: The psychology of clothes. AMS Press, New York (1976)

32. Card, S., Moran, T., Newell, A.: The Model Human Processor: An Engineering Model of Human Performance. In: Boff, K.R., Kaufman, L., Thomas, J.P. (eds.) Handbook of Perception and Human Performance, vol. 2, pp. 1-35. Wiley, New York (1986)

33. Caplan, D., Waters, G.: Verbal working memory and sentence comprehension. Behavioural And Brain Sciences 22, 77-126 (1999)

34. Lohse, G.: The Role of Working Memory in Graphical Information Processing. Behaviour and Information Technology 16(6), 297-308 (1997)

35. Kosslyn, S.: Graphics and Human Information Processing. American Statistical Association 80(391), 499-512 (1985)

36. Sweller, J.: Cognitive Load During Problem Solving: Effects on Learning. Cognitive Science 12(2), 257-285 (1988)

37. Cadle, J., Paul, D., Turner, P.: Business Analysis Techniques 72 Essential Tools for Success. British Informatics Society Ltd., Chippenham (2010)

38. Markus, M., Majchrzak, A., Gasser, L.: A Design Theory For Systems That Support Emergent Knowledge Processes. MIS Quarterly 26(3), 179-212 (2002)

39. Walls, J., Widmeyer, G., Sawy, O.: Building an Information System Design Theory for Vigilant EIS. Information Systems Research 3(1), 36-59 (1992)

40. Burdea, G., Coiffet, P.: Virtual Reality. Wiley, New York (2003)

41. Kock, N., Danesh, A., Komiak, P.: A discussion and test of a communication flow optimization approach for business process redesign. Knowledge and Process Management 15(1), 72-85 (2008)

42. de Leoni, M., van der Aalst, W.M.P., ter Hofstede, A.H.M.: Visual support for work assignment in process-aware information systems. In: Dumas, M., Reichert, M., Shan, M.C. (eds.) BPM 2008. LNCS, vol. 5240, pp. 67-83. Springer, Heidelberg (2008)

43. Zhao, X., Liu, C., Sadiq, W., Kowalkiewicz, M.: Process view derivation and composition in a dynamic collaboration environment. In: Meersman, R., Tari, Z. (eds.) OTM 2008, Part I. LNCS, vol. 5331, pp. 82-99. Springer, Heidelberg (2008)

44. Koschmider, A., Reijers, H., Dijkman, R.: Empirical Support for the Usefulness of Personalized Process Model Views. In: Christian, D., Field, M.,S. (eds.) Computer Science Industry Multi Conference, MKWI 2012 (2012)

45. Kolb, J., Kammerer, K., Reichert, M.: Updatable Process Views for User-Centered Adaption of Large Process Models. In: Liu, C., Ludwig, H., Toumani, F., Yu, Q. (eds.) Service Oriented Computing. LNCS, vol. 7636, pp. 484-498. Springer, Heidelberg (2012)

46. Jarvis, S., de Freitas, S.: Evaluation of an immersive learning programme to support triage training. In: Games and Virtual Worlds for Serious Applications, VS-GAMES 2009, pp. 117-122 (2009)

47. Dev, P., Youngblood, P., LeRoy Heinrichs, W., Kusumoto, L.: Virtual worlds and team training. Anesth Clin. 25, 321-336 (2007)

48. Dale, E.: Audiovisual methods in teaching. Holt, Rinehart and Winston, New York (1969)

49. S.M., Meyer, M., Munzner, T.: Design Study Methodology: Reflections from the Trenches and the Stacks. IEEE Trans. Visualization and Computer Graphics 18(12), 2431-2440 (2012) 